\title{
LENT score in prognostic assessment of malignant pleural effusion - the impact of target therapy
}

\author{
S. Sousa (iD), J. Caldeira (D), A. Figueiredo (iD), F. Barata (iD \\ Serviço de Pneumologia, Centro Hospitalar e Universitário de Coimbra \\ sofiasousa091@gmail.com
}

\begin{abstract}
Introduction: Malignant pleural effusion (MPE) is a common manifestation in patients with advanced lung cancer. The LENT score was developed as a risk stratification system to predict the survival of these patients. However, following the discovery of molecular markers and a new era of personalized therapy, prognostic estimation became a challenging exercise. The aim of this study was to evaluate the performance of LENT score in predicting MPE survival in EGFR and ALK mutated lung adenocarcinoma.

Methods: A retrospective single-center study of patients with MPE from lung adenocarcinoma followed between January 2008 to December 2018.

Results: Forty-two patients were included in the study (mean age $76.4 \pm 12.6$ years, $52 \%$ female). Of these patients, $29 \%$ exhibited EGFR gene mutation or ALK gene translocation and received tyrosine kinase inhibitor therapy (TKI), in contrast to $71 \%$ of patients without identification of mutational factors and receiving conventional chemotherapy. Based on LENT score, in the sub-group treated with conventional chemotherapy, $67 \%$ had a moderate-risk category and $33 \%$ a high-risk category, with a median overall survival (OS) of 109 (31-406) and 36 (11-77) days, respectively. In the sub-group treated with targeted therapy, $75 \%$ were in a moderate-risk category and $25 \%$ in a high-risk category with a median OS of 1033 (245-1710) and 238 (27-not available) days, respectively. Patients receiving targeted therapy had a longer survival than patients receiving conventional chemotherapy in all LENT score risk categories $(p<0.05)$.

Conclusions: OS in patients with MPE due to lung adenocarcinoma was similar to that predicted by the LENT score, except for patients with EGFR mutation or ALK translocation. In this subgroup, the LENT score seems to underestimate the prognosis. Although this study has limitations regarding sample size, it does reveal, in the present time, some inaccuracy of the LENT score, demonstrating that it needs to be reviewed and revalidated in view of recent therapeutic advances.
\end{abstract}

Keywords: Malignant pleural effusion. LENT score. Adenocarcinoma.

(c) 2021 Grupo de Estudos do Cancro do Pulmão. Published by Publicações Ciência \& Vida. This is an open access article under the CC BY-NC-ND license (http://creativecommons.org/licenses/by-nc-nd/4.0/). 


\section{INTRODUCTION}

Malignant pleural effusion (MPE) is a common manifestation in patients with advanced non-small cell lung cancer (NSCLC). Approximately $15 \%$ of lung cancer patients have a MPE at the time of initial diagnosis, and an even greater proportion demonstrate a MPE at some point in the course of the disease, especially for lung adenocarcinoma histology ${ }^{1-3}$. The overall survival (OS) of NSCLC patients with MPE is approximately 5.5 months after diagnosis ${ }^{4}$. Identifying risk factors that affect prognosis can help these patients with MPE receive optimal individualized treatment.

In 2014, Clive et al. developed the first prognostic model to predict the survival of MPE patients, the LENT score, which is calculated based on pleural fluid LDH (L), Eastern Cooperative Oncology Group (ECOG) performance score (PS) $(E)$, serum neutrophil-to-lymphocyte ratio (NLR) $(\mathrm{N})$ and tumor type $(\mathrm{T})$. It classifies patients into low, moderate, and high-risk groups in what concerns the duration of survival ${ }^{5}$. The LENT score appeared to predict OS significantly better than all other parameters, including ECOG performance status. It is also recommended by the European Respiratory Society clinical practice guidelines in the management of malignant effusions ${ }^{6}$.

However, the LENT score does not take into account the heterogeneity of lung cancer. Moreover, this diversity has expanded with the identification of oncogenic drivers such as epidermal growth factor receptor (EGFR), anaplastic lymphoma kinase (ALK) and c-ROS oncogene 1 (ROS1), as well as the introduction of targeted therapies ${ }^{7-9}$.

The aim of the present study was to evaluate the performance of LENT score in predicting survival in clinical practice in a selected population of MPE secondary to EGFR and ALK mutated lung adenocarcinoma.

\section{METHODS}

\section{Study Population}

We retrospectively selected adult patients with a new and first diagnosis of MPE from adenocarcinoma between January 2008 to December 2018 followed at the Centro Hospitalar e Universitário de Coimbra (CHUC).

\section{Clinical and Physiological Parameters Measurements}

Data were collected regarding age, gender, ECOG status, survival, white blood cell counts, pleural effusion LDH and cytology, and pleural biopsy result. Survival was considered from the date of diagnosis of MPE until the date of death or the date of the last visit. LENT score was calculated for each patient according to the published literature ${ }^{5}$ at the time of diagnosis of MPE and patients classified into risk group (Table 1).

\section{Statistical analysis}

Descriptive statistics was used to describe the characteristics of all the participants. Quantitative data with a normal distribution were expressed as mean and standard deviation (SD) and those variables without normal distribution were expressed as median and interquartile range (P25-P75). Qualitative data were described using relative frequencies. To compare patient's survivals between groups, a variable with non-normal distribution, Mann-Whitney U test were used. All analysis were performed using the statistical program SPSS version 20.0 and all hypothesis tests were bilateral, for a significance level of $5 \%$.

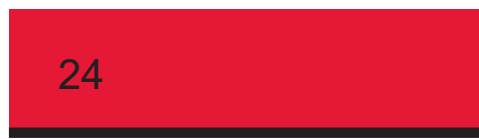


Table 1. The Lent score calculation and prognostic groups ${ }^{5}$

\begin{tabular}{|c|c|c|c|}
\hline \multicolumn{2}{|r|}{ Variable } & Value & Score \\
\hline \multirow{2}{*}{ L } & \multirow{2}{*}{$\begin{array}{l}\text { Pleural fluid LDH } \\
\text { level (IU/L) }\end{array}$} & $<1500$ & 0 \\
\hline & & $>1500$ & 1 \\
\hline \multirow{4}{*}{ E } & \multirow{4}{*}{ ECOG PS } & 0 & 0 \\
\hline & & 1 & 1 \\
\hline & & 2 & 2 \\
\hline & & $3-4$ & 3 \\
\hline \multirow{2}{*}{$\mathbf{N}$} & \multirow{2}{*}{ NLR } & $<9$ & 0 \\
\hline & & $>9$ & 1 \\
\hline \multirow{6}{*}{$\begin{array}{lllllll} & \end{array}$} & \multirow{6}{*}{ Tumor Type } & Mesotelioma & 0 \\
\hline & & $\begin{array}{l}\text { Haematological } \\
\text { malignancy }\end{array}$ & \\
\hline & & Breast cancer & 1 \\
\hline & & Gynaecological cancer & \\
\hline & & Renal cell cancer & \\
\hline & & $\begin{array}{l}\text { Lung cancer } \\
\text { Other tumour types }\end{array}$ & 2 \\
\hline
\end{tabular}

\begin{tabular}{|c|c|c|}
\hline Risk category & Total Score & $\begin{array}{c}\text { Median survival } \\
\text { (range) }\end{array}$ \\
\hline Low risk & $0-1$ & $44(22-77)$ \\
Moderate risk & $2-4$ & $130(47-467)$ \\
Hight risk & $5-7$ & $319(228-549)$ \\
\hline
\end{tabular}

LDH: lactate dehydrogenase; ECOG PS: Eastern Cooperative Oncology Group performance status; NLR: neutrophil lymphocyte ratio.

\section{RESULTS}

A total of 152 patients with MPE secondary to lung adenocarcinoma were identified, but the necessary information of pleural fluid biochemistry could only be obtained in 42 patients, who were included in the present study. The mean age was $76.4 \pm 12.6$ years and $52 \%$ of the patients were female. Concerning the presence of mutational factors, $29 \%$ of the patients exhibited EGFR gene mutation or ALK gene translocation and received targeted therapy with tyrosine kinase inhibitor
Table 2. Demographic and clinical features of patients with MPE from adenocarcinoma lung.

\begin{tabular}{|l|c|}
\hline Variable & $\mathbf{n = 4 2}$ \\
\hline Age, years & $76.4 \pm 12.6$ \\
\hline Gender & $52 \%$ female, 48\% male \\
\hline EGFR mutation & $22 \%$ \\
\hline ALK translocation & $7 \%$ \\
\hline Overall survival, days [median (IQR)] & $151(33-465)$ \\
\hline Treated with target therapy with TKI's & $29 \%$ \\
\hline Treated with conventional chemotherapy & $71 \%$ \\
\hline ECOG status [median (IQR)] & $1(1.0-2.75)$ \\
\hline Pleural fluid LDH [median (IQR)] & $752(522-1545)$ \\
\hline Blood NLR [median (IQR)] & $4,6(3.1-8.6)$ \\
\hline LENT score [median (IQR)] & $4(3-5)$ \\
\hline
\end{tabular}

EGFR: epidermal growth factor receptor; ALK: anaplastic lymphoma kinase; ECOG Eastern Cooperative Oncology Group; TKI's: tyrosine kinase inhibitors; LDH: lactate dehydrogenase; NLR: neutrophil lymphocyte ratio.

therapy (TKI). In contrast, $71.4 \%$ of patients didn't have any identification of mutational factors and received conventional chemotherapy.

The median LENT score in our cohort was 4 (3-5) and the OS was 151 (33-465) days (Table 2). The majority of patients (69\%) belonged to the to moderate-risk category, $31 \%$ to the high-risk category, and none to the low-risk category. The median (IQR) survival for patients in the moderate-risk and high-risk categories was 217 (77-629) and 41 (15-162) days, respectively.

In sub-group analysis of patients treated with targeted therapy, $75 \%$ had a moderate-risk category and $25 \%$ had a high-risk category based on LENT score, with a median (IQR) survival and a 6 months survival rate of 1033 (245-1710) days and $89 \%$, and 238 (27-not available) days and $67 \%$, respectively. In the sub-group of patients treated with conventional chemotherapy, $67 \%$ had 
Table 3. Comparison of median survival based on LENT score between the sub-group of patients receiving target therapy and the subgroup of patients receiving conventional chemotherapy.

\begin{tabular}{|c|c|c|c|}
\hline Risk Category (Lent score) & Patients receiving target therapy & $\begin{array}{l}\text { Patients receiving conventional } \\
\text { chemotherapy }\end{array}$ & $p$ value \\
\hline \multicolumn{4}{|l|}{ Survival days, median (IQR) } \\
\hline High risk (Lent score $\geq 5$ ) & 238 (27-not available) & $36(11-77)$ & $p<0.05^{*}$ \\
\hline Moderate risk (Lent score 2-4) & $1033(245-1710)$ & $109(31-406)$ & $p<0.05^{*}$ \\
\hline Low risk (Lent score 0-1) & - & - & - \\
\hline \multicolumn{4}{|l|}{ Survival rate at 6 months } \\
\hline High risk (Lent score $\geq 5$ ) & $89 \%$ & $45 \%$ & $p<0.05^{*}$ \\
\hline Moderate risk (Lent score 2-4) & $67 \%$ & $10 \%$ & $p<0.05^{*}$ \\
\hline Low risk (Lent score 0-1) & - & - & - \\
\hline
\end{tabular}

a moderate-risk category and $33 \%$ had a high-risk category based on LENT score, with a median (IQR) survival and a 6 months survival rate of 109 (31-406) days and $45 \%$, and 36 (11-77) days and $10 \%$, respectively. Patients receiving target therapy had a longer survival than patients receiving conventional chemotherapy in all LENT score risk categories $(p<0.05)($ Table 3$)$.

\section{DISCUSSION}

In this study, we acknowledged that the survival in patients with MPE from lung adenocarcinoma without an activating EGFR mutation or ALK translocation, and therefore receiving conventional chemotherapy was similar with that predicted by the LENT score. However, the actual survival in those receiving TKl's was higher than predicted by the LENT score. The median survival in those patients in our cohort was 238 days in high-risk category and 1033 days in moderate-risk category, which is better than the survival of 130 days in high-risk category and 319 days in moderate-risk reported by Clive et al. ${ }^{5}$ in their LENT validation cohort (Table 1 and 2).
Similar findings have been published in 2015 by investigators from Japan who reported that $87.5 \%$ of the patients receiving TKI's in the high-risk group based on the LENT score had a survival of more than 6 months ${ }^{10}$. In 2018, Abisheganaden ${ }^{11}$ confirmed that the EGFR mutation was a protective factor for lung adenocarcinoma patients and that LENT score underestimated survival in those patients and probably would need some adjustment in this subgroup.

The identification of oncogenic drivers such as EGFR, ALK, and ROS1 in recent years, and the introduction of targeted therapies, have dramatically improved survival rates ${ }^{9}$. Prior to the advent of TKl's, the median survival used to be 6.5-8 months in patients with MPE from lung cancer ${ }^{12}$. Currently the median survival in those receiving TKI's has been reported as 14 months with an 1 year survival rate of $52.9 \%{ }^{8}$.

Our study has the limitation of being a single center experience with a retrospective observational design and with a limited sample size. Additionally, an information bias might have occurred due to data collection from the patients' medical records. 
In conclusion, LENT score needs to be used with caution in MPE patients with lung adenocarcinoma carrying mutational drivers and treated with TKIs, as it seems to underestimate their survival. The rapid development of new therapies in the recent years has created new prognostic groups among these patients, and LENT score needs to be revised and revalidated to improve its accuracy in this subgroup.

\section{ORCID}

Sofia Rodrigues Sousa (iD) 0000-0001-8043-3874 João Nunes Caldeira (iD) 0000-0001-7949-1946 Ana Figueiredo (ID) 0000-0002-7949-8440 Fernando Barata (ID) 0000-0002-6306-0938

\section{REFERENCES}

1. Agalioti, T., A.D. Giannou, and G.T. Stathopoulos, Pleural involvement in lung cancer. J Thorac Dis, 2015. 7(6): p. 1021-30.

2. Thomas, J.M. and A.I. Musani, Malignant pleural effusions: a review. Clin Chest Med, 2013. 34(3): p. 459-71.

3. Heffner, J.E. and J.S. Klein, Recent advances in the diagnosis and management of malignant pleural effusions. Mayo Clin Proc, 2008. 83(2): p. 235-50.
4. William, W.N., Jr., et al., Revisiting stage IIIB and IV non-small cell lung cancer: analysis of the surveillance, epidemiology, and end results data. Chest, 2009. 136(3): p. 701-709.

5. Clive, A.O., et al., Predicting survival in malignant pleural effusion: development and validation of the LENT prognostic score. Thorax, 2014. 69(12): p. 1098-104.

6. Bibby, A.C., et al., ERS/EACTS statement on the management of malignant pleural effusions. European Respiratory Journal, 2018. 52(1): p. 1800349.

7. Kasapoglu, U.S., et al., Prognostic factors affecting survival in non-small cell lung carcinoma patients with malignant pleural effusions. Clin Respir J, 2016. 10(6): p. 791-799.

8. Wu, S.-G., et al., Survival of lung adenocarcinoma patients with malignant pleural effusion. European Respiratory Journal, 2013. 41(6): p. 1409-1418.

9. Clark, J.W. and D.L. Longo, Recent progress in systemic treatment for lung cancer. Curr Opin Pulm Med, 2018. 24(4): p. 355-366.

10. Takahashi, T., et al., Validation of the LENT score in Japanese sample: The impact of EGFR-TKI. European Respiratory Journal, 2015. 46(suppl 59): p. PA4328.

11. Abisheganaden, J., et al., An Observational Study Evaluating the Performance of LENT Score in the Selected Population of Malignant Pleural Effusion from Lung Adenocarcinoma in Singapore. Respiration, 2018. 96(4): p. 308-313.

12. Sugiura, S., et al., Prognostic value of pleural effusion in patients with non-small cell lung cancer. Clin Cancer Res, 1997. 3(1): p. 47-50. 